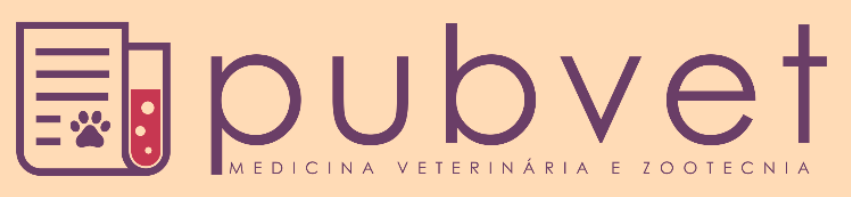

https://doi.org/10.31533/pubvet.v14n5a564.1-7

\title{
Insucessos e complicações no tratamento de dermatite atópica canina em Poodle: relato de caso (2012-2019)
}

\author{
Katherinne Barth Wanis Figueiredo ${ }^{1 \oplus}$, Juliana de Abreu Pereira ${ }^{2 *} \bullet$, Talita Wajczyk ${ }^{10}$, \\ Darlene Luciana Guse Kaiser ${ }^{1}$, Thaís Karoline Pereira ${ }^{10}$ \\ ${ }^{I}$ Discente do curso de Medicina Veterinária da Universidade Sociedade Educacional de Santa Catarina (UNISOCIESC), campus Joinville. \\ Joinville - SC Brasil. \\ ${ }^{2}$ Docente do curso de Medicina Veterinária da UNISOCIESC, campus Joinville. Joinville - SC Brasil. \\ *Autor para correspondência, E-mail: juliana.abreu@unisociesc.com.br
}

\begin{abstract}
Resumo. A dermatite atópica canina (DAC) é uma condição inflamatória pruriginosa baseada em predisposição genética para desenvolver alguma hipersensibilidade a alimentos e / ou alérgenos ambientais, com sinais clínicos em resposta à exposição, caracterizados por coceira intensa. O diagnóstico é feito por anamnese, por exclusão e exames histopatológicos; e o tratamento consiste em controlar os sinais clínicos, evitando a exposição a alérgenos, tanto quanto possível, uma vez que a condição não tem cura. Este relato de caso trata da evolução clínica de um canino da raça poodle afetado por DAC em tratamento no período de sete anos, concomitantemente com complicações devido à pancreatite canina. A melhora clínica foi alcançada após várias tentativas de tratamento, sendo o uso de glicocorticoides a única terapia que atenuou significativamente os sinais clínicos da doença. Com isso, o canino permanece clinicamente controlado para a dermatite atópica com corticosteroide e o uso de xampus e hidratantes específicos, além de redução da exposição a alérgenos ambientais.
\end{abstract}

Palavras chave: alérgenos, cão, pancreatite, tratamento

\section{Failures and complications in the treatment of canine atopic dermatitis in a Poodle: a case report (2012-2019)}

\begin{abstract}
Canine atopic dermatitis (DAC) is an itchy inflammatory condition based on genetic predisposition to develop some hypersensitivity to food and / or environmental allergens, with clinical signs in response to exposure, characterized by severe itching. The diagnosis is made by anamnesis, by exclusion and histopathological exams. The treatment consists of controlling clinical signs, avoiding exposure to allergens as much as possible, since the condition has no cure. This case report deals with the clinical evolution of a canine poodle affected by DAC undergoing treatment over a seven-year period, concomitantly with complications due to canine pancreatitis. Clinical improvement was achieved after several attempts at treatment, the use of glucocorticoids being the only medication that significantly attenuated the clinical signs of the disease. Thus, the canine remains clinically controlled for atopic dermatitis with corticosteroids and the use of specific shampoos and moisturizers, in addition to reducing exposure to environmental allergens.
\end{abstract}

Keywords: Allergens, dog, pancreatitis, treatment

\section{Fracasos y complicaciones en el tratamiento de la dermatitis atópica canina en un Caniche: informe de un caso (2012-2019)}

Resumen. La dermatitis atópica canina (DAC) es una afección inflamatoria con prurito basada en predisposición genética para desarrollar cierta hipersensibilidad contra los 
alérgenos alimentarios y/o ambientales, con signos clínicos en respuesta a la exposición, caracterizados por picazón intensa. El diagnóstico se realiza mediante anamnesis, exclusión y exámenes histopatológicos. Y el tratamiento consiste en controlar los signos clínicos, evitando la exposición a los alérgenos tanto como sea posible, ya que la afección no tiene cura. El presente informe de caso trata sobre la evolución clínica de un canino de la raza Poodle afectado por DAC sometido a tratamiento en el período de siete años, concomitantemente con complicaciones debido a la pancreatitis canina. La mejoría clínica se logró después de varios intentos de tratamiento, siendo el uso de glucocorticoides el único fármaco que atenuó significativamente los signos clínicos de la enfermedad. Con esto, el canino permanece clínicamente controlado para la dermatitis atópica con la terapia con corticosteroides y el uso de champús e hidratantes específicos, así como para reducir la exposición a los alérgenos ambientales.

Palabras clave: alérgenos, perro, pancreatitis, tratamiento

\section{Introdução}

A dermatite atópica canina (DAC) é uma afecção frequente, de aspecto pruriginoso e difícil controle tanto em humanos como em animais (Marsella \& De Benedetto, 2017). Trata-se de uma das enfermidades com reações cutâneas mais comuns na prática clínica de animais de companhia. Consiste em uma afecção inflamatória, normalmente, baseada em uma predisposição genética em desenvolver alguma hipersensibilidade contra alérgenos alimentares e/ou ambientais, o que cursa com sinais clínicos típicos da exposição, como por exemplo: prurido entre as patas; focinho, axila, virilha e orelhas que podem causar lesões devido à automutilação do animal. Além disso, alopecia, vermelhidão e espessamento da pele também são achados comuns (Thorsteinsdottir et al., 2016).

O diagnóstico é feito por anamnese, por exclusão e exames histopatológicos. Diversos são os tratamentos empregados; porém, apenas visando o controle dos sinais (uma vez que não há cura para a DAC) e evitando a exposição a alérgenos tanto quanto possível.

Este relato de caso trata do diagnóstico e evolução clínica de um canino da raça poodle afetado por DAC em tratamento no período de seis anos. São detalhadas as terapêuticas multimodais empregadas, a abordagens às infecções secundárias por malasseziose e dermatofitose que acometeram o paciente assim como o desenvolvimento de pancreatite e lama biliar.

\section{Relato de caso}

Um cão Poodle, fêmea, com peso corporal de $7,2 \mathrm{~kg}$, idade de 14 anos, foi diagnosticado com DAC em 2012. Na anamnese, a proprietária informou que adquiriu o cão com 60 dias de vida, não sabendo informar o histórico familiar do animal. A paciente foi castrada apenas no sexto ano de vida. Era devidamente vacinada, vermifugada e submetida mensalmente à controle de ectoparasitas com fipronil pour on (Frontline Plus ${ }^{\circledR}$ ) para cães até $10 \mathrm{~kg}$.

Um ano após a castração, o animal iniciou quadro de lambedura excessiva da porção distal dos membros torácicos, posteriormente evoluindo para os pélvicos. O cão não possuía convívio com outros animais. A residência era higienizada diariamente com produtos de limpeza não específicos para animais. O animal alimentava-se apenas com ração para cães adultos (Golden ${ }^{\circledR}-120 \mathrm{~g}$ ao dia) e uma vez ao dia recebia petiscos (Biscoito Biscrok Pedigree ${ }^{\circledR}-2$ unidades ao dia). Tinha histórico de tratamentos de otites recorrentes e episódios esporádicos de êmese após início da lambedura dos membros. Os banhos eram realizados uma vez por semana em Pet Shop com xampu neutro específico para animais.

Após um mês do início dos sinais clínicos, o animal foi levado para avaliação sendo observado eritrema e alopecia na parte distal dos membros. Devido ao fato de o cão passar bastante tempo sozinho, de início, acreditou-se na possibilidade de distúrbio comportamental e optou-se pelo tratamento com fluoxetina $1,4 \mathrm{mg} / \mathrm{kg}$ uma vez ao dia durante seis meses. Entretanto, durante o período do tratamento os sinais foram se agravando e o animal passou a apresentar odor fétido e descamação da pele na região das lambeduras. Já com 5 meses de tratamento, diante da falha terapêutica, optou-se por realizar o desmame da fluoxetina e suspender o tratamento. 
Como o animal realizava controle regular de ectoparasitas, não foi cogitado dermatite alérgica à picada de ectoparasitas, mas sim a possibilidade de dermatite alérgica de contato. Sendo assim, a proprietária realizou a troca dos produtos de limpeza doméstica por produtos hipoalergênicos, porém o cão persistia com agravo do prurido.

Devido à nova suspeita clínica de DAC foi realizado um exame histopatológico com fragmentos de pele obtidos por biópsia incisional a partir da região distal do membro torácico direito e região rostral da face medindo respectivamente $5 \times 1,5 \times 3 \mathrm{~mm}$ e $2,5 \times 1 \times 2 \mathrm{~mm}$. O laudo histopatológico evidenciou na epiderme; hiperplasia, espongiose e ortoqueratose em trançado de cesto. Na derme superficial havia congestão de vasos sanguíneos, edema e infiltrado inflamatório monomorfonuclear perivascular com mastócitos, linfócitos e plasmócitos. Folículos pilosos, glândulas sebáceas e apócrinas não apresentaram alterações histopatológicas. Não foram evidenciados parasitas foliculares e fungos. Através do exame foi confirmada a suspeita de DAC.

Após diagnóstico optou-se pela exclusão total dos petiscos e troca da ração regular para fórmula hipoalergênica (Hypoallergenic - Royal Canin ${ }^{\circledR}$ - $140 \mathrm{~g}$ ao dia), havendo melhora parcial dos sinais. Após três meses (D90), o animal persistia com eritrema e prurido da pele sendo realizado a troca do shampoo regular para hipoalergênico (Allermyl ${ }^{\circledR}$ Glyco - Virbac) e recomendada a aplicação pós banho de formulações de ômega 3 Spot On (Allerderm ${ }^{\circledR}$ Spot - On - Virbac), sem evidências de melhora clínica. Diante da automutilação do animal, optou-se por iniciar por via oral maleato de dexclorfeniramina 0,27 $\mathrm{mg} / \mathrm{kg}$ a cada doze horas por quinze dias.

Com a introdução do anti-histamínico ao tratamento, o animal obteve melhora significativa dos sinais em uma semana após o início do antialérgico, todavia após seis meses o prurido e o eritrema se estenderam para o corpo do animal havendo uma maior automutilação. Sendo assim foi optado por iniciar por via oral prednisona $1,38 \mathrm{mg} / \mathrm{kg}$ a cada vinte e quatro horas por sete dias e cefalexina 20,8 $\mathrm{mg} / \mathrm{kg}$ a cada doze horas por dez dias. Após o término da medicação o animal ainda apresentava crises de prurido com intensa automutilação, sendo optado por manter o corticóide três vezes na semana e fazer o desmame conforme redução dos sinais.

Depois de um ano e meio de tratamento, o animal iniciou quadro de êmese com restos alimentares, abdome distendido, dor abdominal e prostração. A paciente foi levada imediatamente para avaliação onde foram realizados exames de imagem, laboratoriais e bioquímicos séricos conforme apresentado na tabela 1 .

Tabela 1. Achados bioquímicos séricos e de imagem no episódio de pancreatite aguda da paciente. Valores de referência conforme Kaneko et al. (2008)

\begin{tabular}{lcc}
\hline Parâmetro & Resultado & Referência \\
\hline Glicose, mg/dL & 237 & $65-118$ \\
Amilase, U/L & 1714 & $185-700$ \\
Lipase, U/L & 2018 & $13-200$ \\
ALT, U/L & 30 & $21-102$ \\
AST, U/L & 10 & $23-66$ \\
Fosfatase alcalina, U/L & 290 & $20-156$ \\
Ultrassonografia & Pâncreas com dimensões aumentadas, contornos irregulares e pouco definidos, peritonite difusa
\end{tabular}

As elevações de amilase, lipase, glicemia e fosfatase alcalina, juntamente com os achados no exame de imagem e os sinais clínicos, foram essenciais para o diagnóstico de pancreatite aguda grave.

Diante do quadro, o animal permaneceu internado por 7 dias, foi submetido à jejum de 48 horas e tratado com antibiótico (ceftriaxona EV 35mg/kg a cada 12 horas), analgésico (tramadol EV $2 \mathrm{mg} / \mathrm{kg}$ a cada 8 horas), antiemético (metocloropramida $0,2 \mathrm{mg} / \mathrm{kg}$ a cada 8 horas) e fluidoterapia (solução fisiológica $0,9 \%$ via endovenosa - EV - em infusão contínua na taxa de $24 \mathrm{ml} / \mathrm{h}$ ).

Após a melhora do quadro de pancreatite, optou-se pela alteração da ração hipoalergênica para um alimento com baixo teor de gordura (Gastrointestinal Low Fat ${ }^{\circledR}$ - Royal Canin - $160 \mathrm{~g}$ ao dia). Após quinze dias da mudança da dieta foi realizado novo ultrassom, o qual evidenciou melhora do quadro de inflamação. Alguns meses após a internação, conforme pode ser visto na Figura 1, o cão apresentou piora importante do quadro de dermatite (prurido, eritrema, automutilação, irritabilidade e odor fétido), 
sendo cada vez menos responsiva a medicações. Os sinais eram minimizados apenas com o uso de corticoides e em doses elevadas. Durante a consulta foi realizado um raspado cutâneo o qual constatouse que o animal estava apresentando um quadro de malasseziose em todo o corpo.
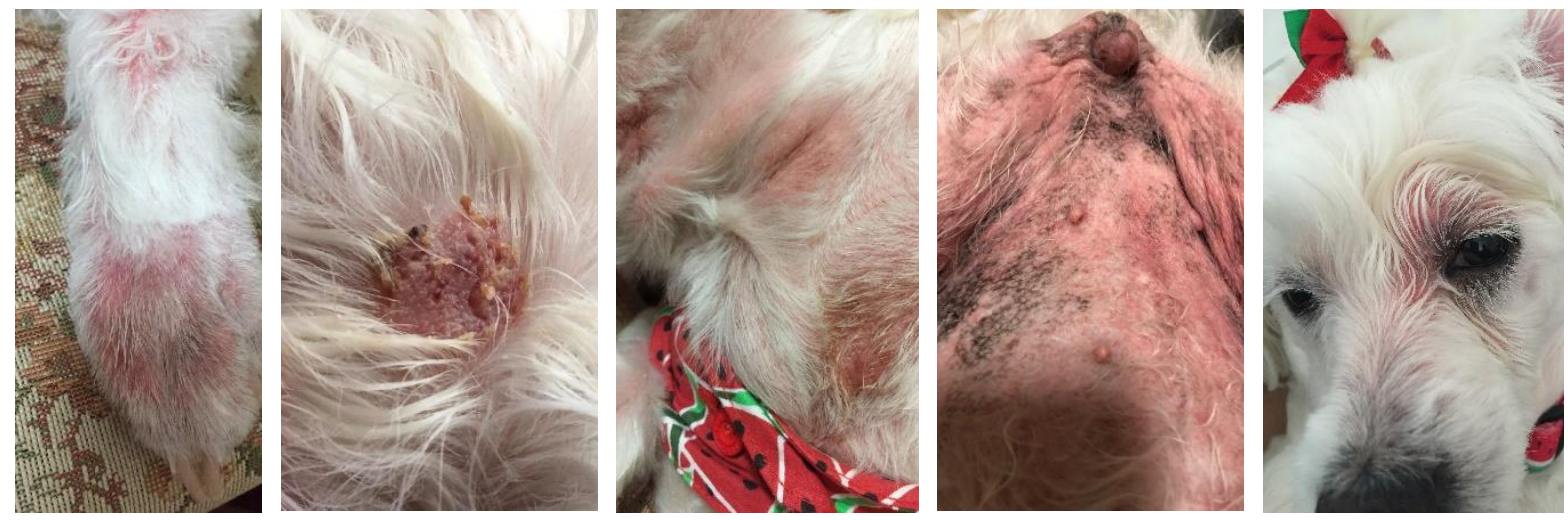

Figura 1. Aspectos das lesões cutâneas apresentadas pela paciente no período descrito.

Diante dos insucessos de tratamento, optou-se por encaminhá-lo ao dermatologista veterinário, onde foi prescrito itraconazol manipulado da dose de $8,3 \mathrm{mg} / \mathrm{kg}$ a cada vinte e quatro horas por quinze dias, prednisona $1,38 \mathrm{mg} / \mathrm{kg}$ a cada vinte e quatro horas por sete dias, cloridrato de hidroxizina $1,73 \mathrm{mg} / \mathrm{kg}$ a cada doze horas por trinta dias. Além disso, foi alterada a ração para uma formulação específica para animais com pele sensível (Skin Care ${ }^{\circledR}$ - Royal Canin - 120 g ao dia). Durante o uso das medicações o animal apresentou melhora importante do quadro clínico, porém após um mês voltou a apresentar êmese de restos alimentares sendo optado por retornar à ração com baixos teores de gordura (Gastrointestinal Low Fat ${ }^{\circledR}$ - Royal Canin). Ao término das medicações prescritas pelo dermatologista, o cão voltava a apresentar todos os sinais da dermatite, causando muito incômodo ao mesmo e automutilação, bem como frustração do proprietário. Por opção deste, não foram realizadas as tentativas de tratamento com dieta natural, imunoterapia com vacinas e uso de fármacos imunossupressores.

O proprietário então, diante do insucesso do tratamento, optou por suspender o acompanhamento com o dermatologista e retornou apenas para o clínico veterinário. Após alguns anos de tratamentos com anti-inflamatórios, antialérgicos, antimicrobianos e corticoides, apenas este último realizava uma remissão dos sinais da dermatite. Já apresentando diversas lesões, alopecia, hiperpigmentação e descamação da pele; e sendo medicado com dose elevada $(2,7 \mathrm{mg} / \mathrm{kg})$ de corticoide, o animal voltou a apresentar sinais clínicos compatíveis com pancreatite; sendo levado à clínica veterinária onde foi realizado o mesmo protocolo diagnóstico da internação anterior. Assim, diante dos resultados dos exames contidos na tabela 2 , o cão foi diagnosticado com pancreatite crônica.

Tabela 2. Achados bioquímicos séricos e de imagem no episódio de pancreatite crônica da paciente. Valores de referência conforme Kaneko et al. (2008)

\begin{tabular}{lcc}
\hline Parâmetro & Resultado & Referência \\
\hline Glicose, $\mathrm{mg} / \mathrm{dL}$ & 360 & $65-118$ \\
Amilase, U/L & 1517 & $185-700$ \\
Lipase, U/L & 1820 & $13-200$ \\
Ultrassonografia & Pâncreas com dimensões aumentadas, contorno pouco definido, ecogenicidade mista e textura grosseira
\end{tabular}

Diante da enfermidade, foi realizada nova internação, optando-se por realizar o mesmo tratamento da internação anterior. Após 7 dias houve a remissão dos sinais e o animal foi liberado para retornar ao domicílio juntamente com o proprietário.

Durante os anos seguintes foram tentados diversos novos tratamentos conforme surgiam no mercado, a citar shampoo e spray à base de fitosfignosina (Douxo Calm ${ }^{\circledR}$ - Ceva), shampoos com diferentes formulações e princípios ativos $\left(\right.$ Cortishamp $^{\circledR}$ - Dr. Clean, Cloresten ${ }^{\circledR}$ - Dr. Clean, Peroxydex Spherulites ${ }^{\circledR}$ - Virbac, Sebolytic Spherulites ${ }^{\circledR}$ - Virbac, Dermogen ${ }^{\circledR}$ - Agener), pomadas (Quadriderm ${ }^{\circledR}$ Schering Plough, Crema $6 \mathrm{~A}^{\circledR}$ - Labyes), sprays (óleo de melaleuca 5\%), além de medicamentos 
injetáveis em casos de automutilação grave, porém sem melhora significativa dos sinais clínicos. No ano de 2016, o animal iniciou episódios frequentes de êmese, sendo levado novamente para consulta veterinária. Para investigação do quadro foi realizada ultrassonografia abdominal e exames bioquímicos séricos conforme apresentado na tabela 3.

Tabela 3. Achados bioquímicos séricos e de imagem no episódio de lama biliar da paciente. Valores de referência conforme Kaneko et al. (2008)

\begin{tabular}{lcc}
\hline Parâmetros & Resultado & Referência \\
\hline Colesterol, mg/dL & 275 & $135-270$ \\
Triglicerídeos, mg/dL & 1069 & $20-112$ \\
Lipase, U/L & 2018 & $13-200$ \\
ALT, U/L & 119 & $21-102$ \\
Fosfatase alcalina, U/L & 633 & $20-156$ \\
Ultrassonografia & Vesícula biliar com paredes finas e ecogênicas, apresentando acúmulo de sedimento em \\
\hline
\end{tabular}

No laboratório verificou-se que o soro se encontrava acentuadamente lipêmico, o que dificultou a realização das provas bioquímicas. Diante dos resultados liberados, juntamente com o exame de imagem e os sinais clínicos o cão foi diagnosticado com lama biliar. Para tratamento em domicílio foi prescrito bezafibrato $2,77 \mathrm{mg} / \mathrm{kg}$ e ácido ursodesoxicólico $9,7 \mathrm{mg} / \mathrm{kg}$ sendo administrados cada um a cada 24 horas por 60 dias, sendo recomendada a repetição dos exames após trinta dias de início do tratamento. Durante esse período houve piora do quadro de dermatopatia.

No início do ano de 2017, após uma grave lesão por automutilação, foram realizados exames bioquímicos hormonais devido à suspeita de associação da DAC a hipotireoidismo, entretanto todos os exames realizados estavam dentro dos parâmetros normais (T4 livre pelo método de radioimunoensaio $1,09 \mathrm{ng} / \mathrm{dl}$; TSH RIE 0,18 ng/dl). Diante disso, foi optado por iniciar tratamento com oclacitinib 3,6 mg (Apoquel ${ }^{\circledR}$ ) sendo prescrito um comprimido a cada 24 horas por 15 dias. Entretanto, não houve melhora dos sinais clínicos de prurido e automutilação. Durante uma consulta posterior de rotina foi optado por iniciar suplementação com biotina $10 \mathrm{mg}$ uma vez ao dia durante três meses, porém o animal também não apresentou melhora do quadro dermatológicos.

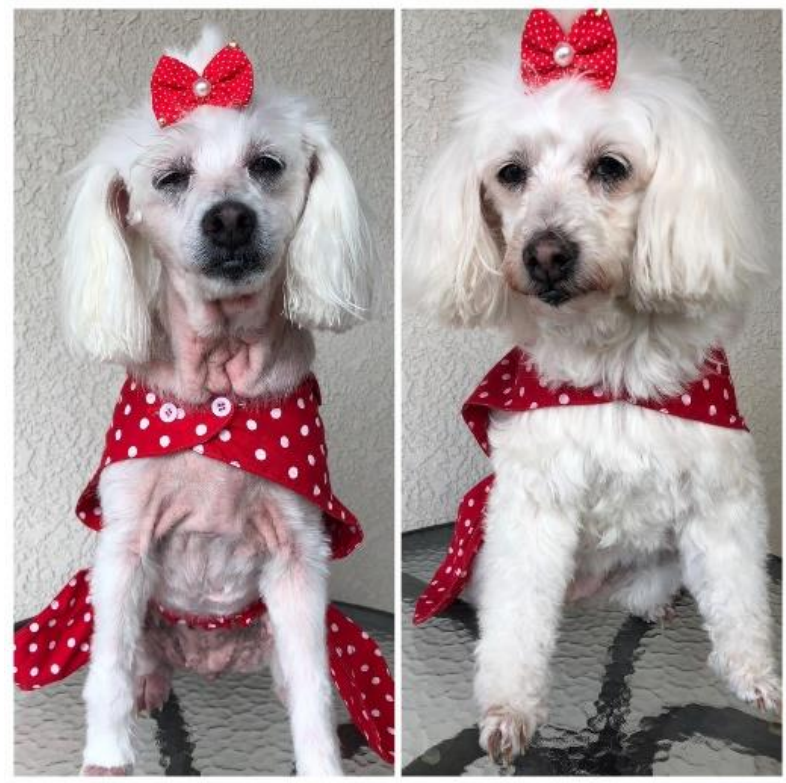

Figura 2. Aspecto da pele e da pelagem após a estabilização do quadro de DAC.

Ao final do ano de 2017, foi iniciado novo tratamento com deflazacort $1,5 \mathrm{mg}$ sendo prescrita uma cápsula a cada 24 horas por 6 dias e após esse período deveria ser mantido o medicamento a cada 48 horas conforme ocorrência dos sinais. Com esse tratamento o animal apresentou melhora importante do prurido; e consequentemente do eritrema, da descamação cutânea, do odor fétido e falhas de pelagem. 
Até o início de 2018, o animal possuia sinais de dermatite controlados, sendo mantido o protocolo terapêutico utilizando deflazacort $1,5 \mathrm{mg}$ uma cápsula a cada 5 dias ou conforme sinais clínicos, shampoo hipoalergênico (Allermyl ${ }^{\circledR}$ Glyco), formulações hidratantes spot on contendo ômega 3 (Allerderm ${ }^{\circledR}$ Spot-On) e ração com baixos teores de gordura (Gastrointestinal Low Fat ${ }^{\circledR}$ - Royal Canin $160 \mathrm{~g}$ ao dia). Posteriormente, houve piora do quadro clínico, havendo presença importante de crostas e descamação, hipotricose e importante colonização da pele por Malassezia. Mesmo mantendo o tratamento anterior não se obtinha mais controle do quadro clínico.

Em 2019, a paciente manifestou quadro de dermatofitose, com lesões localizadas na região do pescoço. Foi instituído tratamento tópico com shampoo à base de cetoconazol e clorexidine (Cetodine shampoo), com banhos a cada 3 dias durante um período de 30 dias. Com este tratamento pode-se observar a remissão do quadro de dermatofitose, bem como a resolução por completo do quadro de malasseziose. Após o controle da levedura, pode-se observar o crescimento do pelo, sendo completamente restabelecida a pelagem em todas as áreas onde havia hipotricose. Conforme pode ser observado na Figura 2, atualmente a paciente encontra-se controlada da DAC e das infecções secundárias, permanecendo com prurido estimado em grau 2 a 3; com pelagem totalmente restaurada; sem crostas, descamação ou eritema.

\section{Discussão}

A DAC é uma enfermidade clínica que está associada a sinais característicos de uma reação de hipersensibilidade do tipo I, a qual é mediada principalmente pela imunoglobulina E (Marsella \& De Benedetto, 2017). Há evidências crescentes de que existe alguma disfunção na barreira cutânea de animais atópicos (Santoro et al., 2015). Alguns estudos relatam que a disfunção se dá principalmente na diminuição das ceramidas, alterações na expressão da filagrina e maior expressão das enzimas envolvidas no metabolismo da filagrina e perda de água transepidérmica aumentada (Fanton et al., 2017; Marsella et al., 2016).

Tais fatores contribuem para que o animal acometido apresente sinais clínicos importantes, como o prurido, o eritema e a lambedura. Todavia, segundo Favrot (2015) estes não são sinais patognomônicos desta enfermidade. Na fase inicial da DAC, o animal acometido apresentou todos estes sinais, principalmente na porção distal dos membros. Posteriormente ao agravo dos sinais clínicos iniciais, o cão apresentou uma infecção secundária por Malassezia sp, o que vem de encontro ao descrito por Nuttal (2010), os quais relatam em seu estudo que, devido a ocorrência de aliterações na barreira epidérmica de animais com DAC, frequentemente ocorre infecção secundária por Malassezia sp ou Staphylococcus sp.

No presente trabalho, observou-se que o animal iniciou com os sinais clínicos já com idade avançada, e um ano após a castração. Segundo estudo de Sundburg et al. (2016) cães castrados apresentam alto risco para DAC; porém, as fêmeas possuem mais riscos que os machos, o que vem de encontro ao sexo do animal do presente relato.

Para o controle da DAC, é necessário uma terapêutica multimodal que abranja a eliminação dos agentes alérgenos, o reforço da barreira cutânea, utilização de fármacos anti-inflamatórios sistêmicos e tópicos, imunoterapia específica, terapêutica antimicrobiana, dentre outros (Hnilica \& Medleau, 2012). A terapêutica com o glicocorticoide foi o único tratamento que surtiu efeito satisfatório no animal; porém, ter seu uso prolongado cursa com efeitos adversos como a pancreatite (Viana, 2014). Sinais de pancreatite aguda surgiram mesmo não excedendo o limite terapêutico do fármaco conforme dose sugerida por Viana (2014). Quando foi necessário elevar as doses ultrapassando o limite sugerido, a pancreatite evoluiu de aguda para crônica. A injuria pancreática pode ocorrer devido ao teor gorduroso da alimentação uma vez que triglicerídeos são metabolizados nos capilares dessa glândula (Ettinger et al., 2002; Fossum, 2014) ou ainda pelo uso de glicocorticoides, pois elevam a viscosidade das secreções e potencialmente reduzem o lúmen do ducto pancreático (Bistner et al., 2002).

Atualmente, mesmo após as diversas tentativas de manejo do quadro e complicações clínicas, o animal persiste realizando o controle da DAC com glicocorticoides, por este ser o único fármaco que atenua os sinais clínicos da enfermidade; bem como pela negativa do proprietário em utilizar outros fármacos imunossupressores como protocolo terapêutico. Tal fato vem de encontro ao descrito por 
Rondelli et al. (2015), o qual relata em seu estudo que os animais tratados com corticoterapia sistêmica apresentaram controle satisfatório (> 60\%) dos sinais clínicos da DAC. Todavia, o paciente pode apresentar sinais de poliúria, polidipsia, polifagia, hipertensão arterial, hiperglicemia, trombocitose e glicosúria ao fazer uso por período prolongado (Jericó et al., 2015).

\section{Considerações finais}

A DAC é uma patologia multifatorial que possui grande influência na qualidade de vida do animal e aumenta o risco de infecções secundárias. O protocolo mais eficaz para propiciar alívio do prurido inclui o uso de corticóides, mas com prejuízos à longo prazo para o paciente, sendo necessária a identificação de novas terapias que causem menos efeitos adversos.

\section{Referências bibliográficas}

Bistner, S. I., Ford, R. B., Raffe, M. R., \& Oliveira, P. M. A. (2002). Manual de procedimentos veterinários \& tratamento emergencial. Roca.

Ettinger, S. J., Fedlman, E. C., \& Taibo, R. A. (2002). Tratado de medicina interna veterinaria: enfermedades del perro y el gato. Manole.

Fanton, N., Santoro, D., Cornegliani, L., \& Marsella, R. (2017). Increased filaggrin-metabolizing enzyme activity in atopic skin: a pilot study using a canine model of atopic dermatitis. Veterinary Dermatology, 28(5), 479-e111. https://doi.org/https://doi.org/10.1111/vde.12443.

Favrot, C. (2015). Clinical signs and diagnosis of canine atopic dermatitis. Zurich Open Repository and Archives Animal Breeding, 19(3), 219-222.

Fossum, T. W. (2014). Cirurgia de pequenos animais (4th ed., Vol. 1). Elsevier Brasil.

Hnilica, K. A., \& Medleau, L. (2012). Dermatologia de pequenos animais: atlas colorido e guia terapêutico. Roca.

Jericó, M. M., Kogika, M. M., \& Andrade Neto, J. P. (2015). Tratado de medicina interna de cães e gatos. Guanabara Koogan.

Kaneko, J. J., Harvey, J. W., \& Bruss, M. L. (2008). Clinical biochemistry of domestic animals. Academic press.

Marsella, R., \& De Benedetto, A. (2017). Atopic dermatitis in animals and people: an update and comparative review. Veterinary Sciences, 4(3), 37. https://doi.org/https://doi.org/10.3390/vetsci4030037.

Marsella, R., Papastavros, V., Ahrens, K., \& Santoro, D. (2016). Decreased expression of caspase-14 in an experimental model of canine atopic dermatitis. The Veterinary Journal, 209, 201-203.

Nuttal, T. (2010). Enfermedades cutaneas del perro y el gato. Grupoasis, Zaragoza, Espanha.

Rondelli, M. C. H., Palácios Junior, R. J. G., Oliveira, F. E., \& Costa, M. T. (2015). Estudo retrospectivo da resposta clínica de cães atópicos a diferentes protocolos terapêuticos. INVESTIGAÇÃO, 14(2), 45-50.

Santoro, D., Marsella, R., Pucheu-Haston, C. M., Eisenschenk, M. N. C., Nuttall, T., \& Bizikova, P. (2015). Pathogenesis of canine atopic dermatitis: skin barrier and host-micro-organism interaction. Veterinary Dermatology, 26(2), 84-e25. https://doi.org/https://doi.org/10.1111/vde.12197.

Sundburg, C. R., Belanger, J. M., Bannasch, D. L., Famula, T. R., \& Oberbauer, A. M. (2016). Gonadectomy effects on the risk of immune disorders in the dog: a retrospective study. BMC Veterinary Research, 12(1), 278. https://doi.org/http://dx.doi.org/10.1186/s12917-016-0911-5.

Thorsteinsdottir, S., Thyssen, J. P., Stokholm, J., Vissing, N. H., Waage, J., \& Bisgaard, H. (2016). Domestic dog exposure at birth reduces the incidence of atopic dermatitis. Allergy, 71(12), 1736-1744. https://doi.org/https://doi.org/10.1111/all.12980.

Viana, F. A. B. (2014). Guia terapêutico veterinário. In Lagoa Santa.

Recebido: 17 de fevereiro, 2020.

Aprovado: 21 de março, 2020.

Disponível online: 4 junho, 2020.
Licenciamento: Este artigo é publicado na modalidade Acesso Aberto sob a licença Creative Commons Atribuição 4.0 (CC-BY 4.0), a qual permite uso irrestrito, distribuição, reprodução em qualquer meio, desde que o autor e a fonte sejam devidamente creditados. 